\title{
Transcription factors and miRNAs that regulate fetal to adult CFTR expression change are new targets for cystic fibrosis
}

\author{
Victoria Viart ${ }^{1,2,3}$, Anne Bergougnoux ${ }^{1,3}$, Jennifer Bonini ${ }^{1,2}$, Jessica Varilh ${ }^{1,3}$, \\ Raphaël Chiron ${ }^{4}$, Olivier Tabary ${ }^{5,6}$, Nicolas Molinari ${ }^{7,8}$, Mireille Claustres $^{1,2,3}$ \\ and Magali Taulan-Cadars ${ }^{1,2}$
}

\begin{abstract}
Affiliations: ${ }^{1}$ INSERM U827, Laboratoire de Génétique de Maladies Rares, Montpellier, France. ${ }^{2}$ Université Montpellier I, UFR de Médecine, Montpellier, France. ${ }^{3}$ Laboratoire de Génétique Moléculaire, CHU Montpellier, Montpellier, France. ${ }^{4}$ Centre de Ressources et de Compétences de la Mucoviscidose (CRCM), CHU Montpellier, Montpellier, France. ${ }^{5}$ CDR St Antoine, INSERM UMR-S 938, Paris, France. ${ }^{6}$ UPMC Université Paris 06, Paris, France. ${ }^{7}$ Département d’Information Médicale, CHU Montpellier, Montpellier, France. ${ }^{8}$ UMR 729 MISTEA, Université Montpellier I, Montpellier, France.
\end{abstract}

Correspondence: Magali Taulan-Cadars, INSERM U827, Laboratoire de Génétique de Maladies Rares, Montpellier, France. E-mail: magali.taulandinserm.fr

ABSTRACT The CFTR gene displays a tightly regulated tissue-specific and temporal expression. Mutations in this gene cause cystic fibrosis (CF). In this study we wanted to identify trans-regulatory elements responsible for CFTR differential expression in fetal and adult lung, and to determine the importance of inhibitory motifs in the CFTR-3'UTR with the aim of developing new tools for the correction of disease-causing mutations within CFTR.

We show that lung development-specific transcription factors (FOXA, C/EBP) and microRNAs (miR-101, miR-145, miR-384) regulate the switch from strong fetal to very low CFTR expression after birth. By using miRNome profiling and gene reporter assays, we found that miR-101 and miR-145 are specifically upregulated in adult lung and that miR-101 directly acts on its cognate site in the CFTR-3'UTR in combination with an overlapping AU-rich element. We then designed miRNA-binding blocker oligonucleotides (MBBOs) to prevent binding of several miRNAs to the CFTR-3'UTR and tested them in primary human nasal epithelial cells from healthy individuals and CF patients carrying the p.Phe508del CFTR mutation. These MBBOs rescued CFTR channel activity by increasing CFTR mRNA and protein levels.

Our data offer new understanding of the control of the CFTR gene regulation and new putative correctors for cystic fibrosis.

@ERSpublications

Transcription factors/miRNAs that regulate fetal to adult CFTR expression change are new targets for CF treatment http://ow.ly/zEgYQ

For editorial comments see Eur Respir J 2014; 45: 18-20 [DOI: 10.1183/09031936.00138914].

This article has online supplementary material available from erj.ersjournals.com

Received: May 192014 | Accepted after revision: July 072014 | First published online: Sept 032014

Conflict of interest: Disclosures can be found alongside the online version of this article at erj.ersjournals.com

Copyright OERS 2015 


\section{Introduction}

Most transcription factors are part of multi-protein complexes that recruit other transcription factors and cofactors to mediate local and long-range chromatin changes through physical modifications [1]. Within the transcriptional and post-transcriptional trans-regulatory elements that make up gene regulatory networks, transcription factors often interact with microRNAs (miRNAs) to control gene expression $[2,3]$. miRNAs are post-transcriptional regulators that are expressed in a tissue-specific or developmental stage-specific manner, thereby greatly contributing to cell/tissue-specific protein expression profiles, including during lung organogenesis $[4,5]$.

Mutations in the cystic fibrosis transmembrane conductance regulator (CFTR; MIM\# 602421) gene are responsible for cystic fibrosis (MIM\# 219700), a common recessive lethal disorder. Chronic lung disease is the major cause of mortality and morbidity in patients with cystic fibrosis. CFTR gene expression is spatially and temporally regulated and several studies have demonstrated the differential use of transcription start sites, depending on the tissue type or the developmental stage [6-9]. In the lung, CFTR transcripts can be detected early during embryo development (12th week of pregnancy) and their level progressively increases up to the 24th week of pregnancy. Thereafter, CFTR expression in the airways decreases and is repressed until after birth and remains very low during adult life [10-14]. The changes in CFTR protein expression in human fetuses are consistent with CFTR mRNA temporal pattern [14]. This developmental regulated expression suggests that CFTR is involved in lung organogenesis, possibly by participating in the mechanico-sensory process that is essential for the regulation of Wnt/ $\beta$-catenin signalling during lung development [15].

Several repressors and activators have been implicated in the regulation of CFTR transcription [16-22]. For instance, CCAAT-enhancer-binding protein $(\mathrm{C} / \mathrm{EBP}) \delta$ positively regulates $C F T R$ transcription by binding to an inverted CCAAT element in the promoter [16]. In addition, C/EBP $\beta$ binds to a DNase I-hypersensitive site that is present only in tissues expressing CFTR [23] and we recently demonstrated its binding to CFTR minimal promoter [24]. Chromatin structures that facilitate long-range interactions between various regulatory elements cluster specifically to the CFTR promoter exclusively in CFTR expressing cells $[25,26]$. Recently, it was reported that trans-interacting Forkhead Box A (FOXA) factors induce CFTR activation through binding to a key CFTR cis-regulatory element located in introns 10 and 11 [27-29]. CFTR expression is post-transcriptionally regulated also by miRNAs, such as miR-145 and miR-494 [30-32]. Indeed, several miRNAs, including miR-145, are expressed in primary adult human airway epithelial cells where CFTR expression is low [30]. Moreover, increased expression of some miRNAs has been reported in bronchial epithelium and primary culture of airway epithelia from patients with cystic fibrosis compared to healthy controls $[33,34]$.

Nevertheless, the developmental control of CFTR expression is still poorly understood in humans and the identification of cis- and trans-acting factors responsible for this complex spatio-temporal regulation is challenging. The aims of this study were 1) to identify trans-regulatory elements responsible for the differential expression of CFTR in fetal and adult lung; and 2) to determine the importance of inhibitory motifs in the $3^{\prime} \mathrm{UTR}$ of CFTR in order to develop new tools for CFTR mutation correction in patients with cystic fibrosis.

\section{Materials and methods}

Gene reporter vectors, expression plasmid constructs and directed mutagenesis

The pGL3b-CFTR-WT vector has been previously described [35]. The 3'UTR of the CFTR gene (1.7 kb from the termination codon to the poly-adenylation signal) was subcloned in the pGL3-control vector (Promega, Charbonnieres, France) downstream of the Luciferase gene (pGL3C-CFTR-3UTR). The expression plasmids used are listed in supplementary table S1. Point mutations in cis-motifs to abolish binding were introduced by direct mutagenesis using the QuickChange ${ }^{\oplus}$ II site-directed mutagenesis kit (Stratagene, Massy, France). All constructs were verified by direct sequencing.

Short-interfering RNAs (siRNAs), miRNA precursors and miRNA-binding blocker oligonucleotides (MBBOs) are described in supplementary table S2.

\section{Cell culture}

The cell lines and culture media used for this work are described in table 1.

Nasal cells from healthy individuals or patients with cystic fibrosis and homozygous for the p.Phe508del CFTR mutation were obtained by scratching the inferior turbinate epithelium with ASI Rhino-Pro ${ }^{\circ}$ curettes (Arlington Scientific, Springville, UT, USA). All signed informed written consent and this research project received the agreement by the French ethical research committee (ND-RCB 2011-A01520-41). Nasal cells were cultured in an air-liquid interface (ALI) culture system as previously described [37] with 


\begin{tabular}{|c|c|c|c|c|c|c|c|c|}
\hline A549 & ATCC & $\begin{array}{l}\text { Human pulmonary } \\
\text { epithelial cell line }\end{array}$ & Adult & & Cell line & No & $\begin{array}{l}\text { As previously } \\
\text { described [35] }\end{array}$ & $\begin{array}{l}37^{\circ} \mathrm{C} \\
5 \% \mathrm{CO}_{2}\end{array}$ \\
\hline Beas-2B & ATCC & $\begin{array}{l}\text { Human bronchial } \\
\text { epithelial cell line }\end{array}$ & Adult & & Cell line & No & $\begin{array}{l}\text { As previously } \\
\text { described [35] }\end{array}$ & \\
\hline T84 & ATCC & $\begin{array}{l}\text { Human colic } \\
\text { epithelial cell line }\end{array}$ & Adult & & Cell line & No & $\begin{array}{l}\text { As previously } \\
\text { described [35] }\end{array}$ & \\
\hline $\begin{array}{l}\text { Human } \\
\text { whole lung }\end{array}$ & $\begin{array}{l}\text { DV Biologics, } \\
\text { Cambridge } \\
\text { Bioscience }\end{array}$ & $\begin{array}{l}\text { Human whole lung } \\
\text { cells }\end{array}$ & Fetal & $\begin{array}{l}\text { Fetus, second } \\
\text { trimester }\end{array}$ & $\begin{array}{l}\text { Primary } \\
\text { culture }\end{array}$ & Collagen I & $\begin{array}{c}\text { Fibroblast cellutions media } \\
\text { (DV Biologics, Cambridge } \\
\text { Bioscience) }\end{array}$ & \\
\hline 16HBE140- & & $\begin{array}{l}\text { Human pulmonary } \\
\text { epithelial cell line } \\
\qquad(\mathrm{N} / \mathrm{N})\end{array}$ & Adult & & Cell line & No & $\begin{array}{l}\text { As previously } \\
\text { described [36] }\end{array}$ & \\
\hline
\end{tabular}

\#: full details of the providers are as follows. ATCC, Manassas, VA, USA; ScienCell, Carlsbad, CA, USA; Clinisciences, Montrouge, France; DV Biologics, Costa Mesa, CA, USA; Cambridge Bioscience, Cambridge, UK.

minor modifications. All the media used were supplemented with antibiotics and all the supports were coated with collagen I. After 3 weeks of growth in monolayer, 300000 cells per well were plated in collagen I-coated $12 \mathrm{~mm}$ Transwell-Clear ${ }^{\circledR}$ supports, $0.4 \mu \mathrm{m}$ pore size (Corning Inc., Corning, NY, USA). The ALI medium in the upper compartment was removed after confluence and the medium in the lower compartment was changed every 2-3 days. Experiments were performed when epithelial cells were well differentiated by visual inspection (at least 28 days).

\section{Transient transfections}

All the details of the transient transfection assays are in supplementary table S3.

\section{RNA extraction and $R T-q P C R$}

Total RNA was extracted, reverse transcribed and amplified as previously described [38]. miRNAs were purified with the miRNeasy Mini Kit and the RNeasy MinElute Cleanup kit (Qiagen, Courtaboeuf, France). Reverse transcription was performed using $40 \mathrm{ng}$ of miRNA and the miRCURY LNA ${ }^{\mathrm{TM}}$ Universal cDNA Synthesis Kit (EXIQON, Vedbaek, Denmark) and qPCR was performed with a 1:10 dilution of first strand DNA and microRNA LNATM primers specific for each miRNA (EXIQON). The relative expression levels were calculated using the comparative DDCt method with SNORD44 and SNORD48 small nucleolar RNAs as endogenous controls.

\section{Quantitative chromatin immunoprecipitation assays}

Quantitative chromatin immunoprecipitation (Q-ChIP) was carried out as previously described [24]. Purified cross-linked chromatin was immunoprecipitated with $3 \mu \mathrm{g}$ of each antibody (Santa Cruz, Heidelberg, Germany; Clinisciences, Montrouge, France). As a control for nonspecific DNA binding, $3 \mu \mathrm{g}$ of anti-immunoglobulin G antibodies were used (Santa Cruz, Clinisciences). Results were expressed relative to the input signal and to nonspecific immunoprecipitated chromatin.

\section{Electromobility shift assay}

Electromobility shift assay (EMSA) was performed as previously described [17].

\section{Reporter assay}

Cells were harvested $48 \mathrm{~h}$ after transfection and the activity of firefly luciferase and Renilla luciferase was measured using the Dual-Glo Luciferase Assay System (Promega). 
Labelling, detection and analysis for miRNA profiling

Total RNA (100 ng) from A549 and HBEpiC cells was labelled using the Agilent miRNA Complete Labeling and Hybridization Kit (Agilent Technologies, Massy, France) and then hybridised to the Agilent Human miRNA Microarray (V2, Agilent) that contains probes for 723 mature human miRNAs. Arrays were scanned using an Agilent scanner and features were extracted with the Agilent Feature Extraction software (version 10.5.1.1). Expression data were initially normalised to the 75th percentile and then averaged among the groups using the GeneSpring GX (Agilent) software. The Kruskall-Wallis test was used for group comparisons and the Benjamini-Hochberg correction was applied to adjust for multiple comparisons. Unsupervised hierarchical clustering was performed using the GeneSpring GX software.

\section{Western blotting}

Whole proteins were extracted using 1X Laemmli buffer. Proteins were separated on $7 \%$ or $10 \%$ SDS-PAGE gels and transferred to PVDF membrane (Westran ${ }^{\star}$ Clear Signal Whatman; Dominique Dutscher, Issy les Moulineaux, France). Antibodies and concentrations used are described in the captions to the figures.

\section{CFTR activity}

CFTR activity was assessed by iodide-mediated quenching of the halide-sensitive yellow fluorescent protein (YFP), as previously described [36], using the Premo Halide sensor technology (Invitrogen, Villebon sur Yvette, France), $40 \mathrm{~h}$ after incubation with MBBOs or negative control, CFTR conductance was stimulated with an agonist mixture (forskolin, 3-isobutyl-1-methylxanthine, apigenin) for $10 \mathrm{~min}$. Then, CFTR-mediated iodide efflux was measured in each individual well by recording the fluorescence emission continuously (400 ms per point) for $2 \mathrm{~s}$ (baseline) and after addition of $50 \mu \mathrm{L}$ of $140 \mathrm{mM}$ iodide solution.

\section{Statistical analysis}

Q-ChIP, luciferase and RT-qPCR assays were performed at least three times and samples were analysed at least in triplicate. The t-test was employed for paired comparisons using InStat (GraphPad Software, version 3.0, Instat 3 folder). For assessing the effect of MBBOs, the Wilcoxon signed rank test was used with the R software to generate box plots with significances.

\section{Results \\ Transcription factors involved in the regulation of CFTR temporal expression}

To identify the regulatory elements that participate in CFTR downregulation in human lung after birth, we used primary human fetal bronchial epithelial cells (HBEpiC, three primary cultures from three different donors, gestational age in table 1) and whole lung cells as well as the A549 and Beas-2B cell lines (from adult human lung). CFTR transcript level in fetal HBEpiC and whole lung cells was comparable or even higher than in T84 cells, in which CFTR is constitutively expressed (fig. 1a) [39]. Moreover, CFTR expression in HBEpiC and whole lung cells was more than four-fold higher than in A549, Beas-2B cells and in adult lung RNA from healthy individuals. Based on the well-known CFTR expression profile in fetal and adult lung $[12,13]$, we considered that HBEpiC cells are a representative model of fetal lung cells and that CFTR expression in A549 and Beas-2B cells is comparable to that of adult lung.

To identify putative cis-regulatory motifs, by using open-source bioinformatics software we then performed an in silico analysis of the CFTR 5'UTR. We thus focused on the transcription factors FOXA and C/EBP, based on two criteria: 1) their high score in predicted transcription factor binding and 2) their involvement in lung morphogenesis. First, we confirmed FOXA and C/EBP binding to the CFTR minimal promoter by Q-ChIP (fig. 1b). Moreover, EMSA showed that C/EBP $\beta$ and FOXA2 bind directly to the targeted region (fig. 1c).

Next, we investigated the role of these transcription factors in the regulation of CFTR promoter activity. When overexpressed in A549 and Beas-2B cells (adult lung), FOXA1, FOXA2 and C/EBP $\alpha$ had a repressive effect on CFTR transcription (fig. 2a and b). In line with this result, FOXA1, FOXA2 and $C / E B P \alpha$ silencing using specific siRNAs increased CFTR expression (fig. 2a and b). As control, qPCR and western blot showed that endogenous transcription factor levels were strongly reduced in the presence of each specific siRNA (fig. S1). Conversely, in fetal HBEpiC cells, FOXA1 and FOXA2 did not have any effect, while $\mathrm{C} / \mathrm{EBP} \alpha$ strongly induced CFTR transcription (fig. 2c). Finally, C/EBP $\beta$ overexpression increased endogenous CFTR transcript level in A549 $(2 \times)$ and Beas-2B $(3 \times)$ cells and even more in HBEpiC cells $(30 \times)$ (fig. $2 \mathrm{a}-\mathrm{c}$ ). Analysis of the endogenous level of each transcription factor showed a homogeneous expression in the different cell lines (fig. S2). 
a)

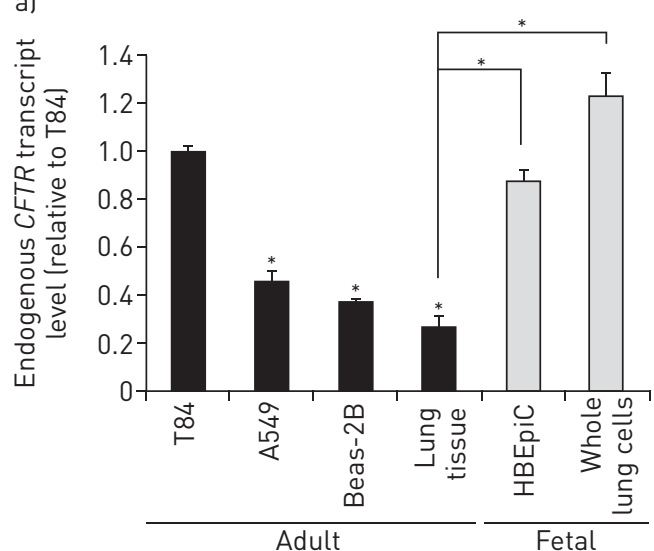

b)

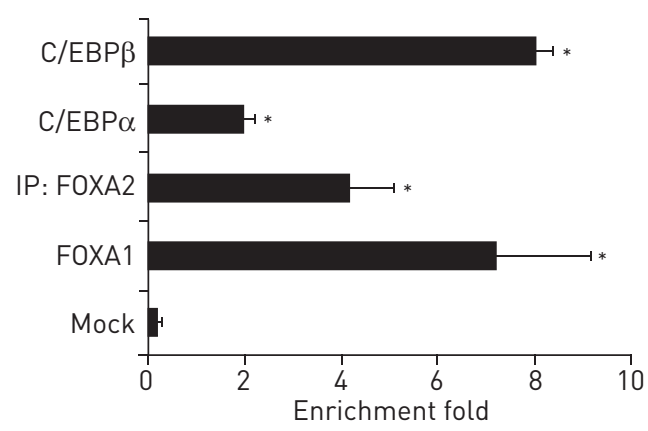

c)

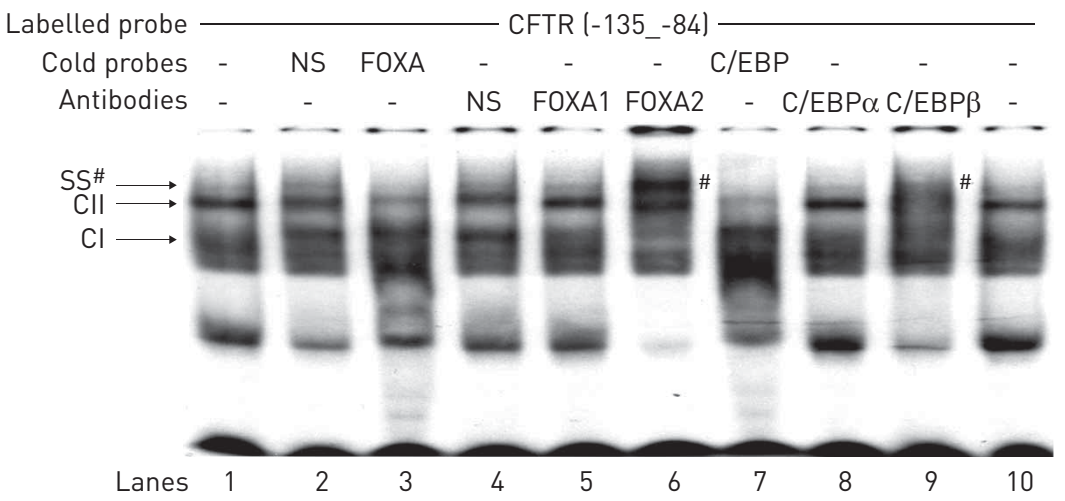

FIGURE 1 C/EBP and FOXA transcription factors bind to the CFTR promoter. a) Quantification of endogenous CFTR transcript level in lung cells compared to the T84 cell line by RT-qPCR. Data were normalised to $\beta$-actin transcript level. b) Binding affinity of the FOXA1, FOXA2, C/EBP $\alpha$ and C/EBP $\beta$ transcription factors for the CFTR promoter. Chromatin from A549 cells was immunoprecipitated (IP) with specific antibodies against FOXA1, FOXA2, C/EBP $\alpha$ and C/EBP $\beta$ or with nonspecific antibodies (IP mock). Data were normalised to non-immunoprecipitated chromatin. c) FOXA and C/EBP transcription factors bind to the -135 to -84 bp region of the CFTR promoter in A549 cells. Electromobility shift assay was performed with specific radiolabelled probes (CFTR-135_-84) incubated with A549 nuclear proteins. Specificity was assessed by competition with an excess of (FOXA and C/EBP) cold probes compared to a nonspecific probe (NS). Supershift (SS) assay was performed with specific anti-FOXA1, -FOXA2, -C/EBP $\alpha$ and -C/EBP $\beta$ antibodies compared to a nonspecific antibody (NS). Arrows highlight specific complexes (CI and CII). ${ }^{*}$ : $\mathrm{p}<0.0001$.

As FOXA and C/EBP factors do not act alone, we re-examined the in silico analysis data by using a lower cut-off in order to select other transcription factors that may contribute to CFTR regulation. Possible candidates of this transcription factor network included RREB-1 (the transcription factor with the highest score), several transcription factors with a known effect on CFTR gene expression, such as USF2, SRF and YY1 [17, 18, 35], and transcription factors involved in lung morphogenesis, but with a lower score than C/ EBPs and FOXAs (SOX17, FOXF1 and NKX2.1) [40]. We then used reporter assays to investigate whether these regulatory elements could participate in the temporal regulation of CFTR expression. Co-transfection of the pGL3b-CFTR-WT reporter vector (wild-type minimal CFTR promoter) with the ubiquitously expressed USF2 and SRF or the developmental-specific NKX2.1 induced luciferase activity in adult and fetal lung cells (fig. 2d). A forced expression of YY1 protein caused a strong decrease in reporter activities ( $\sim 50 \%$ of the control luciferase value). Conversely, RREB-1 inhibited CFTR transcriptional activity in the adult pulmonary A549 and Beas-2B cell lines but not in primary fetal HBEpiC cells. Similarly, the activating effect of SOX17 and FOXF1 in fetal HBEpiC cells was reduced or even abolished in A549 and Beas-2B cells.

These results show that FOXA1, FOXA2 (directly) and C/EBP $\alpha$ negatively regulate CFTR transcription in a specific manner in mature lung cells, while C/EBP $\beta$ induces CFTR transcription through direct binding to the promoter, regardless of the temporal stage. Other transcription factors, such as SOX17, RREB-1 and FOXF1, play also a role in the temporal regulation of CFTR expression in fetal and adult lung. 

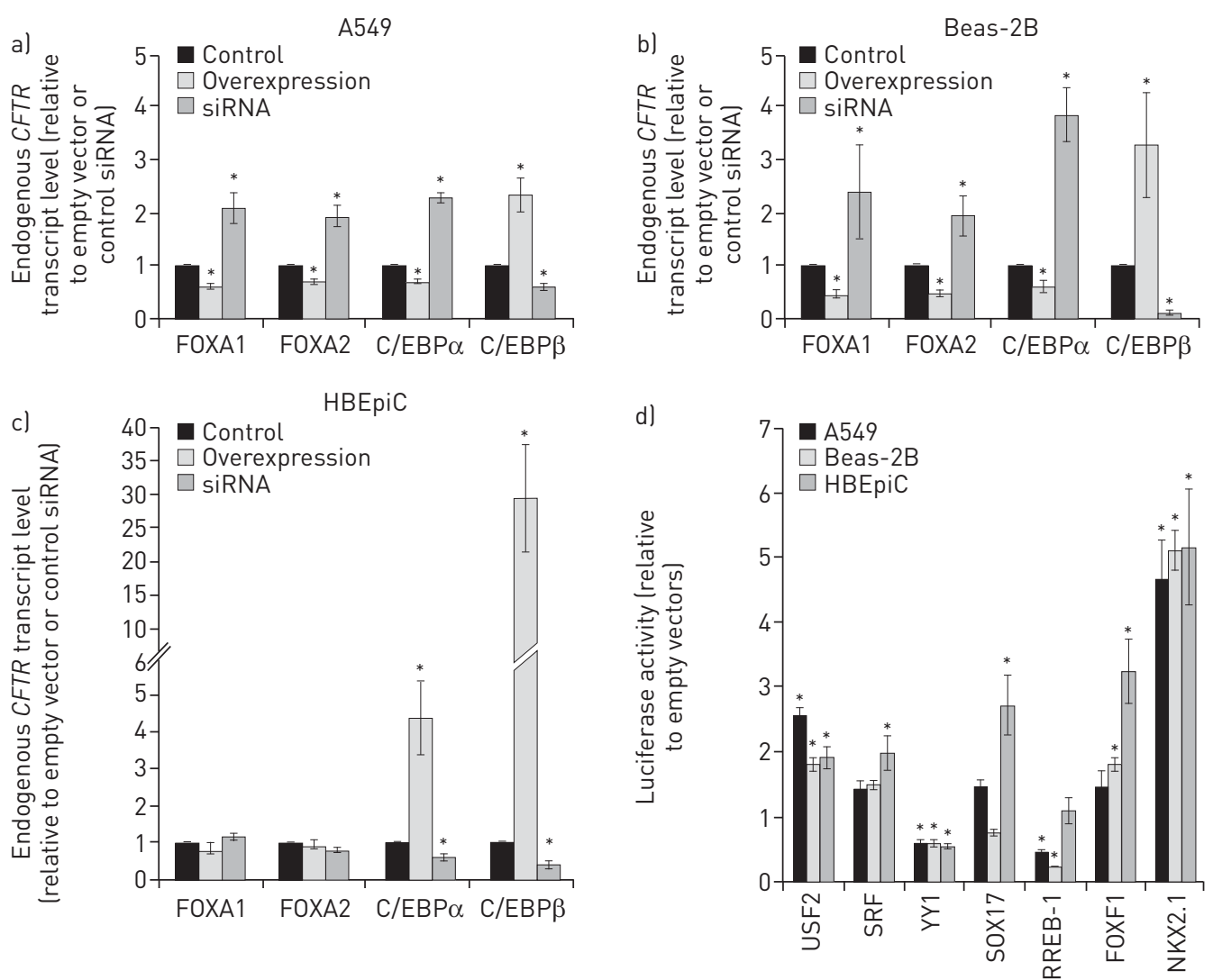

FIGURE 2 Several factors control CFTR transcription. a-c) Effect of FOXA1, FOXA2, C/EBP $\alpha$ or C/EBP $\beta$ overexpression or silencing with specific siRNAs on the endogenous CFTR transcript level in adult pulmonary cells (A549 (a) and Beas-2B cells (b)) and c) fetal cells (HBEpIC cells). RT-qPCR data were normalised to $\beta$-actin transcript level. d) Role of different transcription factors in CFTR transcriptional activity regulation. Luciferase activity was measured in the different pulmonary cell lines following co-transfection of the pGL3b-CFTR-WT reporter vector (wild-type minimal CFTR promoter) and of constructs expressing the different transcription factors. Data were normalised to the luciferase activity level following transfection of empty vector. ${ }^{*}$ : $\mathrm{p}<0.0001$.

\section{A complex pattern of cis- and trans-acting elements in the $3^{\prime}$ UTR of CFTR is involved in the temporal regulation of its expression}

To evaluate the effect of the $3^{\prime} \mathrm{UTR}$ on the post-transcriptional regulation of CFTR, we then transfected A549, Beas-2B and HBEpiC cells with the pGL3C-CFTR-3UTR reporter vector (CFTR 3'UTR) or vector alone. The 3'UTR of CFTR strongly repressed luciferase activity in all cell types, indicating that this region contains cis-repressive elements (fig. 3a). Using the bioinformatic tool AREsite (http://rna.tbi.univie.ac.at/ cgi-bin/AREsite.cgi), we identified four new putative AU-rich elements (ARE) in the 3'UTR of the CFTR gene (ARE-4816, ARE-5533, ARE-5698 and ARE-6074) in addition to those previously described [41] and that we renamed ARE-4585, ARE-4760 and ARE-4891, according to their nucleotide position (fig. 3b). To determine the role of these motifs in the regulation of CFTR expression, we transfected A549, Beas-2B and HBEpiC cells with pGL3C-CFTR-3UTR reporter vectors in which each of these motifs was mutated and then measured luciferase activity. Only ARE-4760 appeared to be implicated in mRNA stabilisation because mutation of this motif was associated with a decrease in luciferase activity compared to cells transfected with pGL3C-CFTR-3UTR (wild-type sequence) (fig. 3b). ARE-4585, ARE-5533, ARE-5698 and ARE-6074 seemed to be involved in mRNA destabilisation in A549 and/or Beas-2B cells, whereas they had no significant effect in HBEpiC cells (fig. 3b). The strongest effect was obtained using ARE-5698, which in silico was identified as the most conserved ARE motif in the CFTR $3^{\prime} \mathrm{UTR}$. Other cis-acting elements might explain the repressive activity of the $3^{\prime} \mathrm{UTR}$ of CFTR in adult cell lines. Computational predictions detected 13 putative miRNA-binding motifs in the CFTR 3'UTR (fig. 3c). Among the previously studied miRNAs, miR-145 has been involved in the regulation of CFTR expression in colonic and pancreatic cell lines [30]. We then assessed the role of miRNAs in the post-transcriptional control of CFTR in pulmonary cells by using luciferase reporter assays after transfection with miRNA precursors and the pGL3C-CFTR-3UTR reporter vector. MiR-942, miR-665, miR-383, miR-1290 and miR-1246 did not induce any significant effect in any cell type, whereas miR-600 reduced luciferase activity in all cell lines 

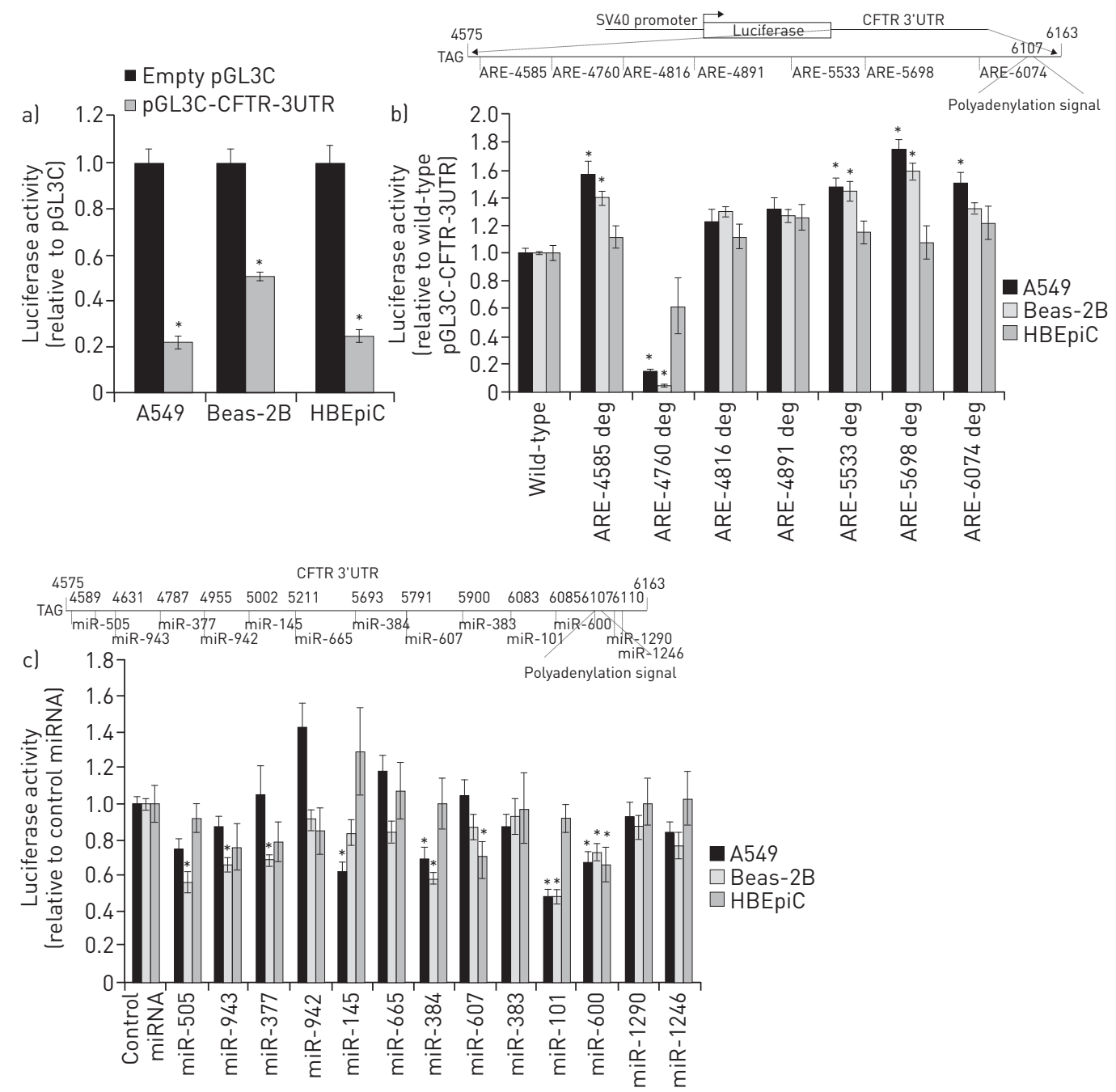

FIGURE 3 Cis- and trans-acting elements in the 3'UTR are involved in the temporal regulation of CFTR gene expression. a) Role of the CFTR $3^{\prime} \mathrm{UTR}$. Luciferase activity was measured in the different lung cell lines after transfection of the reporter vector containing the 3'UTR of the CFTR gene downstream of the Luciferase gene (pGL3C-CFTR-3UTR). Data were normalised to those obtained with empty pGL3C vector. b) Importance of AU-rich element (ARE) motifs in the post-transcriptional regulation of CFTR expression. Each putative ARE site within the CFTR 3'UTR in the pGL3C-CFTR-3UTR vector was mutated and then the effect of their mutation was evaluated by measuring luciferase activity in the different pulmonary cell lines. Data were normalised to the values obtained with the wild-type reporter vector (pGL3C-CFTR-3UTR). c) Role of miRNAs in the post-transcriptional regulation of CFTR expression. Precursors of the miRNAs predicted to bind to the CFTR 3'UTR were transfected in the different pulmonary cell lines and then luciferase activity was measured. Data were normalised to the LNA ${ }^{\mathrm{TM}}$ miRNA inhibitor negative control oligonucleotide (control miRNA; EXIQON, Vedbaek, Denmark). *: $p<0.0001$.

compared to control miRNA (fig. 3c). MiR-505, miR-943, miR-377, miR-145, miR-384 and miR-101 decreased luciferase activity in A549 and/or Beas-2B cells, but not in HBEpiC cells (fig. 3c).

As the strongest repressive effect on CFTR post-transcriptional regulation in A549 and Beas-2B cells was induced by miR-101, we next focused on this miRNA and confirmed its negative impact on endogenous CFTR transcript level after transfection in adult pulmonary cells (fig. 4a). After transfection of the miR-101 precursor, miR-101 overexpression was verified in the three cell lines (fig. $4 \mathrm{~b}$ ). We also confirmed the endogenous expression of miR-101 and its differential expression in A549, Beas-2B (adult) and HBEpiC cells (fetal) (fig. 4c).

Previous studies demonstrated that miRNA-mediated regulation might require the presence of an ARE sequence [42-44]. As the miR-101 and miR-600 binding sites overlap with the ARE-6074 motif and the miR-384 binding site overlaps with the ARE-5698 motif (fig. 4d), we asked whether the effect of these miRNAs following binding to the $3^{\prime} \mathrm{UTR}$ of CFTR is dependent on the integrity of the ARE motifs. To this aim we co-transfected the miR-101, miR-600 and miR-384 precursors with reporter vectors 

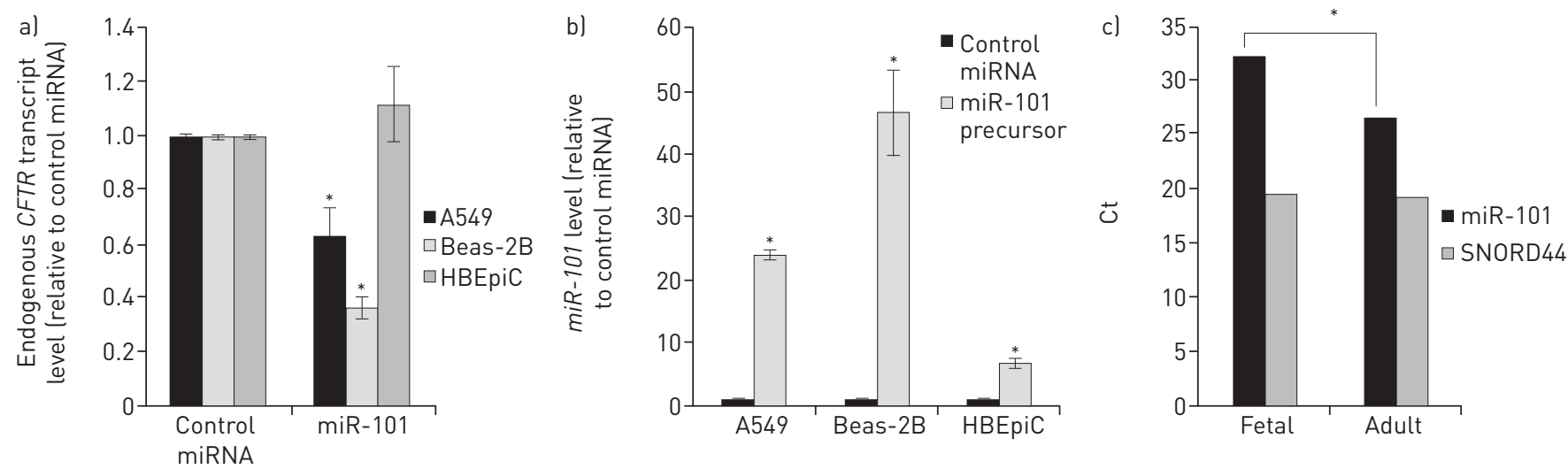

d)

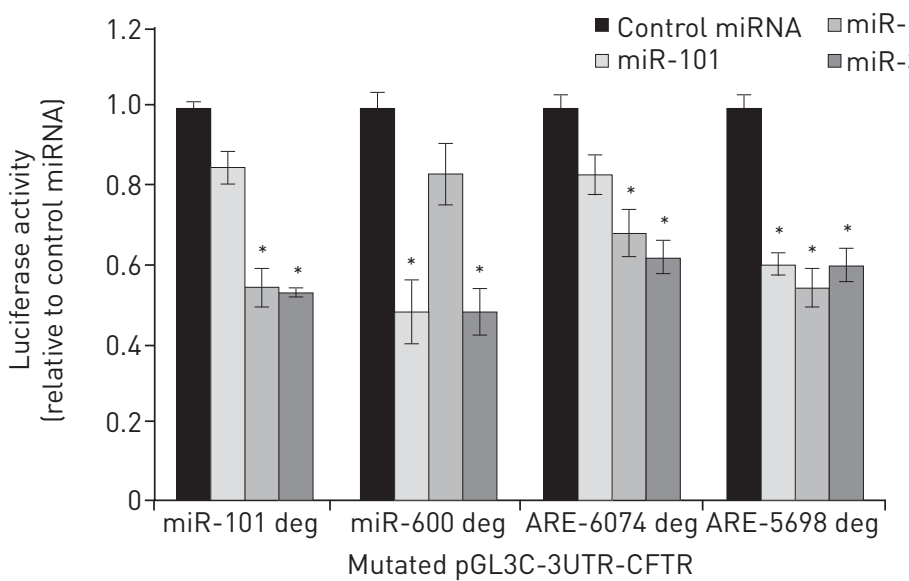

FIGURE 4 Role of miR-101. a) Effect of miR-101 over-expression on the endogenous CFTR transcript level in the different pulmonary cell lines. Data were normalised to $\beta$-actin transcript level. b) Control of miR-101 over-expression in the three cell lines. Hsa-miR-101 level was quantified by RT-qPCR (EXIQON, Vedbaek, Denmark) after transfection of miR-101 precursor or control miRNA in pulmonary cell lines. Data were normalised to SNORD44 transcript level. c) Endogenous miR-101 expression level in fetal (HBEpiC) and adult (A549) lung cells. Data were compared to the expression of the internal control SNORD44. d) Overlapping AU-rich element (ARE) and miRNA binding sites in CFTR 3'UTR. AREs are represented by white squares, miRNA binding sites by grey squares. "\#: mutated nucleotides. Involvement of cis-elements in miRNA effect on CFTR post-transcriptional activity. The role of miR-101, miR-600 and miR-384 was evaluated by measuring luciferase activity in A549 cells after co-transfection of each miRNA precursor with a reporter vector containing the CFTR 3'UTR in which the binding site for miR-101 or miR-600 (miR-101-deg and miR-600-deg), or the ARE-5698 or ARE-6074 motif (ARE-5698-deg and ARE-6074-deg) was mutated. Data were normalised to the luciferase activity in cells transfected with control miRNA. *: p<0.0001.

containing wild type or mutated CFTR 3'UTR. Only miR-101 lost its repressive effect on luciferase activity following the mutation of its binding site within the CFTR sequence or abrogation of the ARE-6074 (fig. 4d). Mutation of ARE-6074 and ARE-5698 did not affect the activity of miR-600 and miR-384, respectively.

As miRNAs have been previously described in lung development mainly in mice, we investigated their differential expression in adult human lung tissue and fetal primary whole lung cells using Agilent DNA microarrays. Analysis of the microarray data showed that 65 miRNAs had the highest expression variability between adult and fetal lung. Among the 30 probes with the strongest expression difference between adult and fetal lung (supplementary table S4), we found that miR-451, miR-150 and miR-145 were specifically upregulated in adult lung (fig. 5a). We confirmed the endogenous expression of miR-145 and its differential expression in A549, Beas-2B (adult) and HBEpiC cells (fetal) (fig. 5b).

These data demonstrate the implication of miRNAs in the tightly controlled developmental regulation of CFTR expression and, more particularly, of miR-101 and miR-145, the expression of which is higher in adult than in fetal lung. Moreover, they show that miR-101 directly acts on its cognate site in combination with an overlapping ARE motif.

\section{From identifying crucial regulators of CFTR expression to testing new potential therapeutic tools} for cystic fibrosis

The region encompassing the miR-101 binding site and ARE-6074 is critical for the miR-101 role in the regulation of CFTR expression. Based on this observation, we designed MBBOs to prevent binding of 


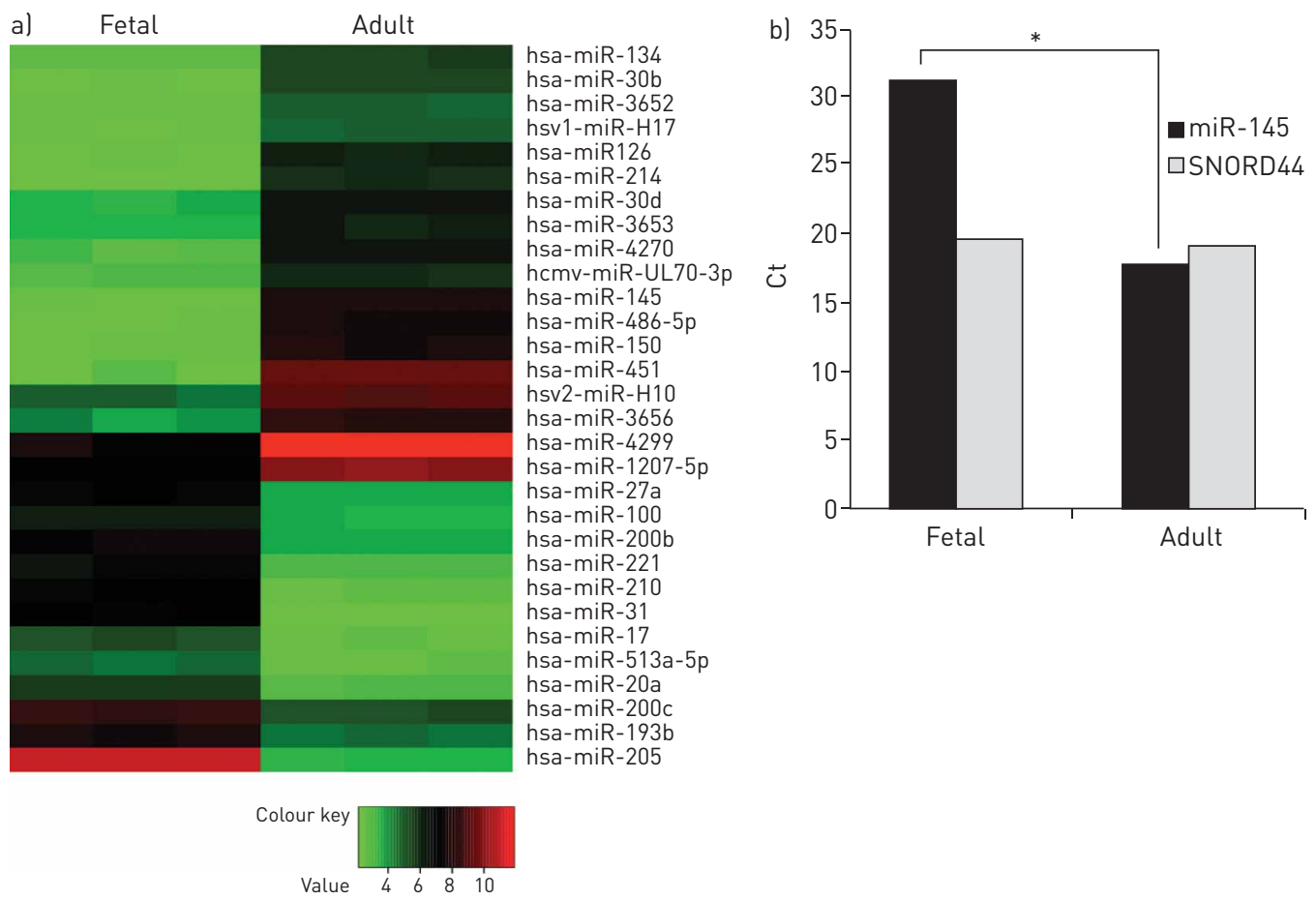

FIGURE 5 miRNA profiling revealed overexpression of miR-145 in adult lung tissues. a) Hierarchical cluster analysis of the miRNAs that are differentially expressed in adult versus fetal lung tissue. Microarray was performed using total miRNA from fetal (HBEpiC) and adult pulmonary (A549) cells. b) Endogenous miR-145 expression level in fetal (HBEpiC) and adult (A549) lung cells. Data were compared to the expression of the internal control SNORD44. *: $\mathrm{p}<0.0001$.

several miRNAs, including miR-101, miR-600, miR-145 and miR-384, to the 3'UTR of CFTR. Co-transfection of these MBBOs with the pGL3C-CFTR-3UTR reporter vector led to a 1.5- to 6-fold increase of luciferase activity in Beas-2B and A549 cells, respectively (fig. 6a). The positive effect on endogenous CFTR expression upon MBBO-1 transfection was confirmed in these cells (fig. 6b).

Next, we evaluated the effect of the MBBOs ex vivo because mutant mice do not develop the characteristic manifestations of human cystic fibrosis. To this aim, we added medium containing control oligonucleotide, MBBO-1 or MBBO-3 without any transfection reagent to the upper compartment of Transwell-Clear ${ }^{\oplus}$ supports in which reconstituted ALI epithelial cells obtained from human nasal cells of control individuals $(\mathrm{n}=8)$ and CF patients homozygous for the p.Phe508del mutation $(\mathrm{n}=6)$ were cultured. After $2 \mathrm{~h}$ at $37^{\circ} \mathrm{C}$, the medium was removed from the upper compartment to restore the ALI. Freshly prepared control oligonucleotide or MBBOs were added every 2 days and CFTR expression was assessed $24 \mathrm{~h}$ post-treatment. MBBO effect was even stronger in epithelial cells from control individuals. Indeed, MBBO-1 induced a 2- to 6-fold increase of the endogenous CFTR expression in the epithelium derived from healthy individuals (fig. 6c) compared to the 2- to 3-fold increase in cells from patients with CF (fig. 6d). This effect was not significantly improved by repeated incubation with MBBO-1.

MBBO-1 and MBBO-3 significantly increased CFTR mRNA (fig. 7a) and protein expression (fig. 7b) in cystic fibrosis epithelia compared to control oligonucleotide. We next investigated MBBO effect on CFTR channel activity by using a functional assay (iodide-mediated quenching of the halide-sensitive YFP variant) and the human bronchial epithelial cell lines CFBE41o- (derived from a patient with cystic fibrosis) and 16HBEo- (normal phenotype). We first confirmed the absence of CFTR-dependent anion transport in CFBE41o- cells (cystic fibrosis) compared to 16HBEo- (non-cystic fibrosis) cells in which iodide entered and quenched YFP fluorescence (fig. 7c). Addition of Inh-172 (a CFTR inhibitor) in non-cystic fibrosis cells led to results comparable to those obtained in cystic fibrosis cells, confirming that the assay measures CFTR-dependent anion transport. Incubation with MBBO-1 and MBBO-3 for $2 \mathrm{~h}$, significantly increased anion transport in cystic fibrosis cells compared to untreated cells (fig. 7d) and fluorescence quenching was proportional to the amount of CFTR detected in the cells by immunoblotting.

These data support the importance of the regions encompassing the miR-101 and miR-145 binding sites in CFTR regulation and suggest that MBBOs could represent a new therapeutic option for CF. 

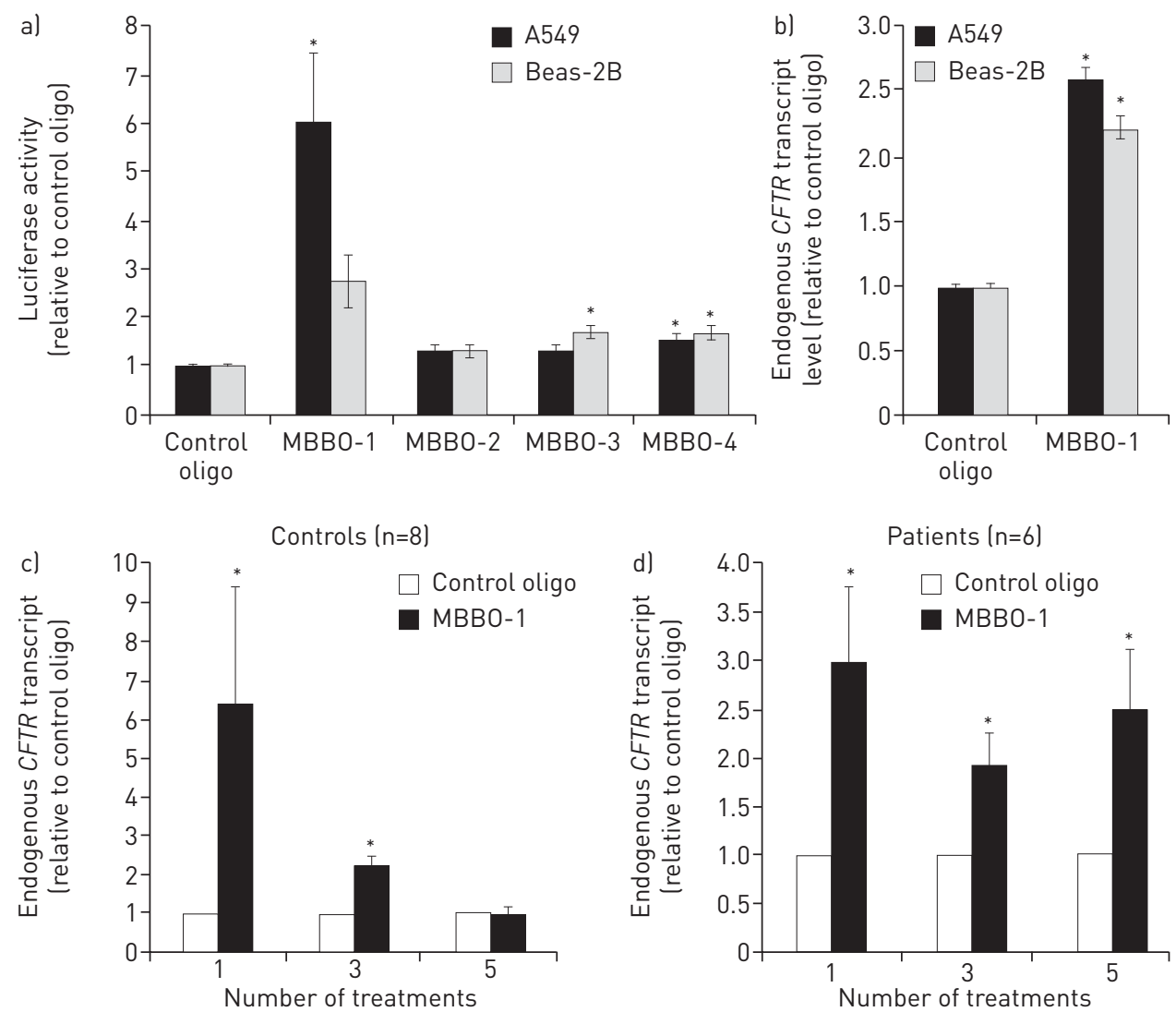

FIGURE 6 Effect of miRNA-binding blocker oligonucleotides (MBBOs) on CFTR expression in pulmonary cells. a) Effect of different MBBOs on CFTR post-transcriptional regulation. MBBOs (100 nM) were transfected together with the pGL3C-CFTR-3UTR reporter vector in A549 and Beas-2B pulmonary cells and luciferase activity data were normalised to miRCURY LNA ${ }^{\mathrm{TM}}$ microRNA Inhibitor Negative Control (control oligonucleotide; EXIQON, Vedbaek, Denmark). b) Effect of MBBO-1 on the endogenous CFTR transcript level in A549 and Beas-2B pulmonary cells. CFTR mRNA level was assessed by RT-qPCR following the transfection of MBBO-1 or control oligonucleotide. Data were normalised to $\beta$-actin transcript level. c, d) Effect of MBBO-1 on the endogenous CFTR transcript level in nasal epithelial cells from c) healthy controls $(n=8)$, or from d) CF patients homozygous for the p.Phe508del mutation $(n=6)$. CFTR mRNA level was assessed by RT-qPCR $24 \mathrm{~h}$ after one, three or five treatments with MBBO-1 or control oligonucleotide. Data were normalised to $\beta$-actin transcript level. *: $\mathrm{p}<0.0001$.

\section{Discussion}

Expression studies carried out in humans, mice and goats have revealed that the CFTR gene is developmentally regulated $[10,12-14,45]$. The most well-known site of developmentally regulated CFTR expression is the airway surface epithelium, with relatively high expression during embryonic and fetal development, followed by a marked decrease in expression after birth [45]. Despite extensive studies, the mechanisms accounting for this switch in CFTR expression remain unknown.

In a critical region of the CFTR gene that contains several naturally occurring variants $[18,35,46]$, we found many ubiquitous [17, 18] and tissue- or lung developmental-specific transcription factors [40] involved in coordinating the switch from strong to very low CFTR expression in lungs after birth. The specific occupancy of these factors on the promoter may, in interaction with others factors, including FOXA1 and C/EBP $\beta$ that bind to other part of the CFTR gene depending on the nucleosome positioning [28], influence the particular pattern of expression of this gene. We also show that miRNAs, including miR-101 and miR-145, negatively regulate the level of CFTR transcripts in adult lung cells, whilst having no effect in fetal lung cells. In addition to its specific role in mature lung cells, miR-101 decreases luciferase activity in an embryonic kidney cell line [31], whereas it does not affect CFTR mRNA stability in pancreatic cell lines [30], suggesting a potential role as a tissue-specific factor. We then demonstrate the implication of miRNAs in the tightly controlled developmental regulation of CFTR expression and more particularly we show that miR-101 acts on its cognate site in combination with an overlapping ARE motif.

Finally, we demonstrate the benefit of characterising regulatory factors to identify novel therapeutic targets. Early studies indicated that complementation of just $6-10 \%$ of CFTR transcripts generate enough CFTR 

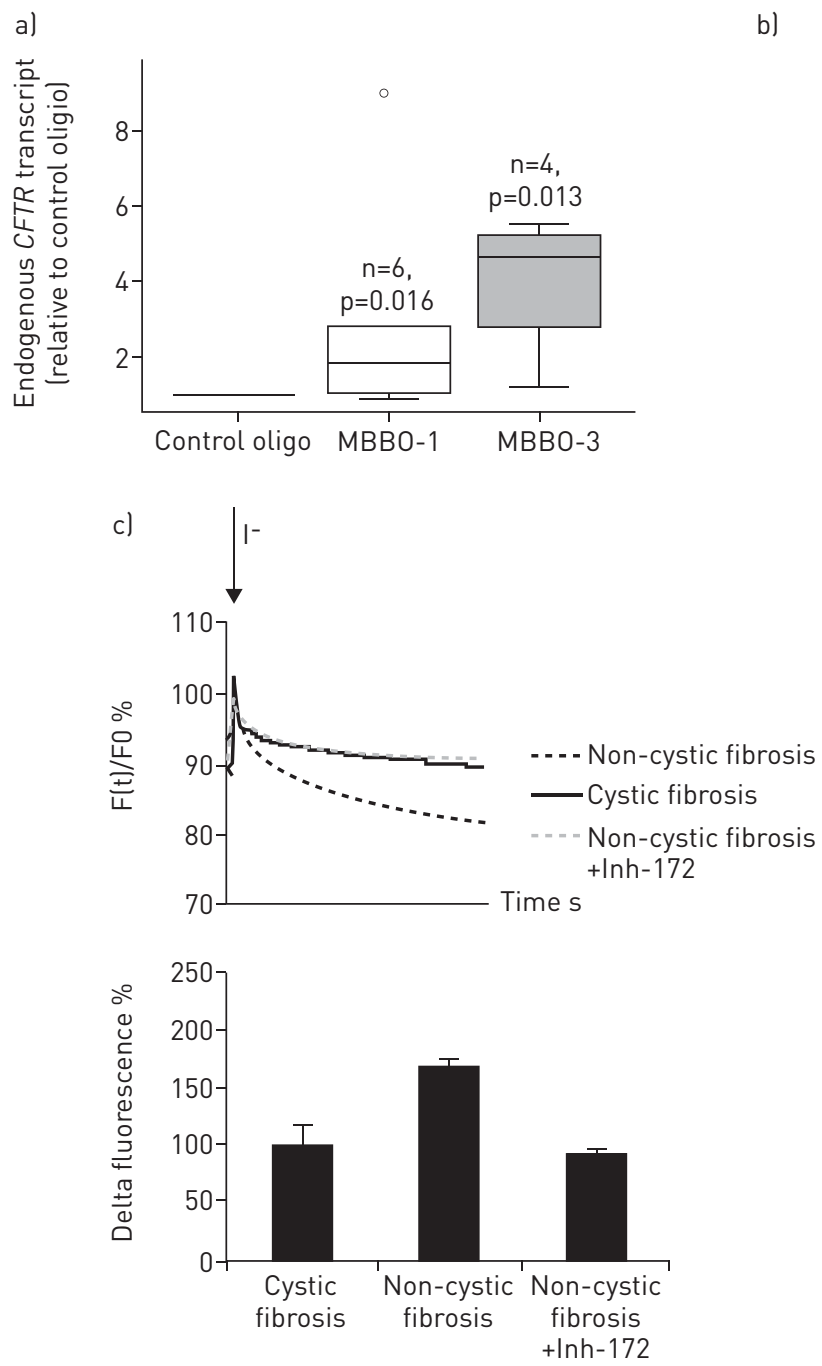

b)

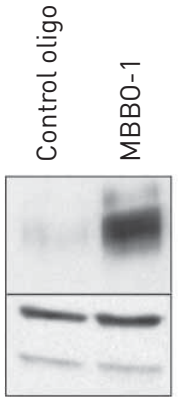

Patient 1

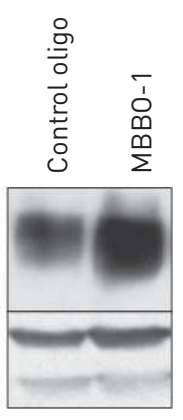

Patient 2

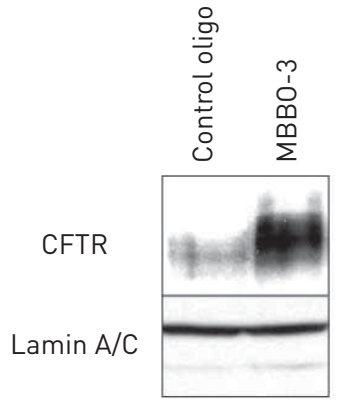

Patient 1

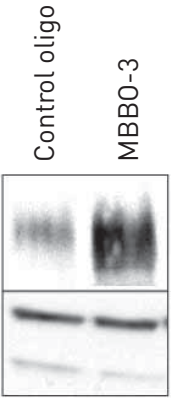

Patient 2
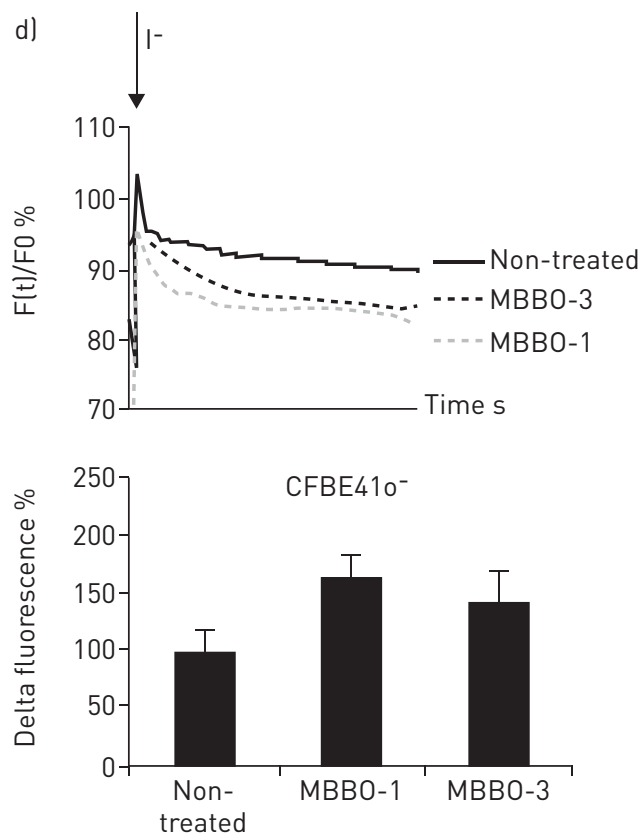

FIGURE 7 Effect of miRNA-binding blocker oligonucleotides (MBBOs) on CFTR expression and activity in epithelial cells from cystic fibrosis (CF) patients. a) Effect of MBBO-1 and MBBO-3 on endogenous CFTR transcript level in nasal epithelial cells from CF patients who are homozygous for the p.Phe508del mutation ( $24 \mathrm{~h}$ after the first treatment with $100 \mathrm{nM}$ MBBO-1, MBBO-3 or control oligonucleotide at $37^{\circ} \mathrm{C}$ for $2 \mathrm{~h}$ ). Data were normalised to $\beta$-actin transcript level. b) Effect of MBBO-1 and MBBO-3 on endogenous CFTR protein level in nasal epithelial cells from CF patients who are homozygous for the p.Phe508del mutation. Immunoblots were performed with an anti-CFTR antibody (1:400, clone MM13-4; Millipore, Molsheim, France) using total protein extracts prepared $24 \mathrm{~h}$ after the first treatment with MBBO-1, MBBO-3 or control oligonucleotide. Lamin A/C protein level (1:10 000, anti- Lamin A/C antibody; Sigma Aldrich, Saint-Quentin Fallavier, France) was used as loading control. c, d) Representative cell fluorescence recordings from bronchial cells that transiently express halide-sensitive yellow fluorescent protein (YFP) (the scale bar reports the percentage of total cell fluorescence). CFTR activity was measured in c) $16 \mathrm{HBE}$ (non-cystic fibrosis) and CFBE41o- (cystic fibrosis) cells, and in d) non-treated or MBBO-treated CFBE41o- cells. Extracellular addition of iodide (arrow) caused YFP quenching at a rate that is proportional to iodide influx and CFTR activity. Channel opening is signalled by a decrease of the probe fluorescence. Quenching is directly proportional to the chloride efflux. Graphs show the quantification of the obtained data. ${ }^{*}: \mathrm{p}<0.0001$.

levels to maintain normal chloride transport in epithelia [47]. These data are supported by findings that the presence of a naturally occurring sequence variation in the CFTR promoter, in cis of a severe mutation, increases transcription. This can allow the production of enough CFTR protein to reach the apical membrane cells and restore partial CFTR channel function, thus inducing a moderate cystic fibrosis phenotype despite the presence of a severe disease-causing mutation [35]. Similarly, stabilisation of p.Phe508del CFTR protein has been associated with increased p.Phe508del CFTR channel activity [48]. Recent work demonstrated that miR-138 mimics might restores CFTR-Phe508del expression and a functional chloride transport [32]. However, the authors underlined the fact that miR-138 mimics may have undesirable effects because miR-138 targets SIN3, a highly conserved transcriptional repressor which regulates many genes [32]. Over the past four decades, therapies for cystic fibrosis have focused entirely on symptoms to improve the patients' quality of life. The first treatment (VX-770) targeted the basic defect in p.Gly551Asp-CFTR (1.6\% of patients with cystic fibrosis worldwide) [49]. The new molecule VX-809 has been investigated for patients carrying the p.Phe508del CFTR mutation; however, alone no clear improvement has been reported [50] and clinical trials testing the combination of different molecules are 
in progress. Herein, we tested a new putative therapeutic tool that specifically targets the CFTR gene [51]. Focusing on miR-101 and miR-145, we designed MBBOs that target the miRNA binding sites in the CFTR 3'UTR instead of the miRNA itself. This blockage led to the correction of CFTR channel activity through stabilisation of CFTR mRNA and increase in the protein level in nasal epithelial cells from patients homozygous for p.Phe508del, the most frequent CFTR mutation. As miR-101 and miR-145 knock-down is associated with deregulation of epigenetic pathways resulting in cancer progression [52] and lung cancer [53], our approach in which MBBOs block only their binding to their cognate CFTR mRNA motif may have therapeutic benefits by stabilising CFTR transcripts and ultimately providing enough functional protein to improve the patients' phenotype without disturbing other signalling cascades. These findings underline the importance in the continued understanding of pathways that are targeted in the lung after birth, which could ultimately lead to new targets in lung disorders, especially in cystic fibrosis.

\section{Acknowledgements}

This work was supported by grants from the French association Vaincre La Mucoviscidose, the CHU and INSERM. V. Viart and J. Bonini were supported by PhD studentships from Vaincre La Mucoviscidose. The authors thank Isabelle Vachier for her help in obtaining agreement from the French ethical research committee. The authors also thank the investigators mentioned in supplementary table S1 for the gifts of crucial reagents.

\section{References}

1 Higgs DR, Vernimmen D, Hughes J, et al. Using genomics to study how chromatin influences gene expression. Annu Rev Genomics Hum Genet 2007; 8: 299-325.

2 Martinez NJ, Walhout AJ. The interplay between transcription factors and microRNAs in genome-scale regulatory networks. Bioessays 2009; 31: 435-445.

3 Shalgi $\mathrm{R}$, Brosh $\mathrm{R}$, Oren $\mathrm{M}$, et al. Coupling transcriptional and post-transcriptional miRNA regulation in the control of cell fate. Aging (Albany NY) 2009; 1: 762-770.

4 Krol J, Loedige I, Filipowicz W, et al. The widespread regulation of microRNA biogenesis, function and decay. Nat Rev Genet 2010; 11: 597-610.

5 Dong J, Jiang G, Asmann YW, et al. MicroRNA networks in mouse lung organogenesis. PLoS One 2010; 5 : e10854.

6 Chou JL, Rozmahel R, Tsui LC, et al. Characterization of the promoter region of the cystic fibrosis transmembrane conductance regulator gene. J Biol Chem 1991; 266: 24471-24476.

7 Yoshimura K, Nakamura H, Trapnell BC, et al. The cystic fibrosis gene has a "housekeeping"-type promoter and is expressed at low levels in cells of epithelial origin. J Biol Chem 1991; 266: 9140-9144.

8 Koh J, Sferra TJ, Collins FS. Characterization of the cystic fibrosis transmembrane conductance regulator promoter region. Chromatin context and tissue-specificity. J Biol Chem 1993; 268: 15912-15921.

9 White NL, Higgins CF, Trezise AE. Tissue-specific in vivo transcription start sites of the human and murine cystic fibrosis genes. Hum Mol Genet 1998; 7: 363-369.

10 Harris A, Chalkley G, Goodman S, et al. Expression of the cystic fibrosis gene in human development. Development 1991; 113: 305-310.

11 McCray PB Jr, Reenstra WW, Louie E, et al. Expression of CFTR and presence of cAMP-mediated fluid secretion in human fetal lung. Am J Physiol 1992; 262: L472-L481.

12 Trezise AE, Chambers JA, Wardle CJ, et al. Expression of the cystic fibrosis gene in human foetal tissues. Hum Mol Genet 1993; 2: 213-218.

13 Tizzano EF, O'Brodovich H, Chitayat D, et al. Regional expression of CFTR in developing human respiratory tissues. Am J Respir Cell Mol Biol 1994; 10: 355-362.

14 Marcorelles P, Montier T, Gillet D, et al. Evolution of CFTR protein distribution in lung tissue from normal and CF human fetuses. Pediatr Pulmonol 2007; 42: 1032-1040.

15 Cohen JC, Larson JE, Killeen E, et al. CFTR and Wnt/beta-catenin signaling in lung development. BMC Dev Biol 2008; 8: 70.

16 Pittman N, Shue G, LeLeiko NS, et al. Transcription of cystic fibrosis transmembrane conductance regulator requires a CCAAT-like element for both basal and cAMP-mediated regulation. J Biol Chem 1995; 270: 28848-28857.

17 Rene C, Taulan M, Iral F, et al. Binding of serum response factor to cystic fibrosis transmembrane conductance regulator CArG-like elements, as a new potential CFTR transcriptional regulation pathway. Nucleic Acids Res 2005; 33: 5271-5290.

18 Taulan M, Lopez E, Guittard C, et al. First functional polymorphism in CFTR promoter that results in decreased transcriptional activity and Sp1/USF binding. Biochem Biophys Res Commun 2007; 361: 775-781.

19 Lopez E, Viart V, Guittard C, et al. Variants in CFTR untranslated regions are associated with congenital bilateral absence of the vas deferens. J Med Genet 2011; 48: 152-159.

20 Zheng W, Kuhlicke J, Jackel K, et al. Hypoxia inducible factor-1 (HIF-1)-mediated repression of cystic fibrosis transmembrane conductance regulator (CFTR) in the intestinal epithelium. FASEB J 2009; 23: 204-213.

21 Burnight ER, Wang G, McCray PB Jr, et al. Transcriptional targeting in the airway using novel gene regulatory elements. Am J Respir Cell Mol Biol 2012; 47: 227-233.

22 Zhang Z, Leir SH, Harris AImmune mediators regulate CFTR expression through a bifunctional airway-selective enhancer. Mol Cell Biol 2013; 33: 2843-2853.

23 Nuthall HN, Vassaux G, Huxley C, et al. Analysis of a DNase I hypersensitive site located $-20.9 \mathrm{~kb}$ upstream of the CFTR gene. Eur J Biochem 1999; 266: 431-443.

24 Viart VVJ, Lopez E, René C, et al. Phosphorylated C/EBP $\beta$ influences a complex network involving YY1 and USF2 in lung epithelial cells. PLoS One 2013:8: e60211.

25 Gheldof N, Smith EM, Tabuchi TM, et al. Cell-type-specific long-range looping interactions identify distant regulatory elements of the CFTR gene. Nucleic Acids Res 2010; 38: 4325-4336. 
Blackledge NP, Ott CJ, Gillen AE, et al. An insulator element $3^{\prime}$ to the CFTR gene binds CTCF and reveals an active chromatin hub in primary cells. Nucleic Acids Res 2009; 37: 1086-1094

Kerschner JL, Harris A. Transcriptional networks driving enhancer function in the CFTR gene. Biochem J 2012; 446: 203-212.

Yigit E, Bischof JM, Zhang Z, et al. Nucleosome mapping across the CFTR locus identifies novel regulatory factors. Nucleic Acids Res 2013; 41: 2857-2868.

Kerschner JL, Gosalia N, Leir SH, et al. Chromatin remodeling mediated by the FOXA1/A2 transcription factors activates CFTR expression in intestinal epithelial cells. Epigenetics 2014; 9: 557-565.

Gillen AE, Gosalia N, Leir SH, et al. MicroRNA regulation of expression of the cystic fibrosis transmembrane conductance regulator gene. Biochem J 2011; 438: 25-32.

Megiorni F, Cialfi S, Dominici C, et al. Synergistic post-transcriptional regulation of the Cystic Fibrosis Transmembrane conductance Regulator (CFTR) by miR-101 and miR-494 specific binding. PLoS One 2011; 6: e26601.

Ramachandran S, Karp PH, Jiang P, et al. A microRNA network regulates expression and biosynthesis of wild-type and DeltaF508 mutant cystic fibrosis transmembrane conductance regulator. Proc Natl Acad Sci USA 2012; 109: 13362-13367.

Oglesby IK, Chotirmall SH, McElvaney NG, et al. Regulation of cystic fibrosis transmembrane conductance regulator by microRNA-145, -223, and -494 is altered in DeltaF508 cystic fibrosis airway epithelium. J Immunol 2013; 190: 3354-3362.

4 Ramachandran S, Karp PH, Osterhaus SR, et al. Post-transcriptional regulation of CFTR expression and function by microRNAs. Am J Respir Cell Mol Biol 2013; 49: 544-551.

5 Romey MC, Pallares-Ruiz N, Mange A, et al. A naturally occurring sequence variation that creates a YY1 element is associated with increased cystic fibrosis transmembrane conductance regulator gene expression. J Biol Chem 2000; 275: 3561-3567.

Saint-Criq V, Ruffin M, Rebeyrol C, et al. Azithromycin fails to reduce inflammation in cystic fibrosis airway epithelial cells. Eur J Pharmacol 2012; 674: 1-6.

Gras D, Bourdin A, Vachier I, et al. An ex vivo model of severe asthma using reconstituted human bronchial epithelium. J Allergy Clin Immunol 2012; 129: 1259-1266.

Viart V, Des Georges M, Claustres M, et al. Functional analysis of a promoter variant identified in the CFTR gene in cis of a frameshift mutation. Eur J Hum Genet 2012; 20: 180-184.

Riordan JR, Rommens JM, Kerem B, et al. Identification of the cystic fibrosis gene: cloning and characterization of complementary DNA. Science 1989; 245: 1066-1073.

Maeda Y, Dave V, Whitsett JA. Transcriptional control of lung morphogenesis. Physiol Rev 2007; 87: $219-244$.

Baudouin-Legros M, Hinzpeter A, Jaulmes A, et al. Cell-specific posttranscriptional regulation of CFTR gene expression via influence of MAPK cascades on 3'UTR part of transcripts. Am J Physiol Cell Physiol 2005; 289: C1240-C1250.

Jing Q, Huang S, Guth S, et al. Involvement of microRNA in AU-rich element-mediated mRNA instability. Cell 2005; 120: 623-634.

Sun G, Li H, Rossi JJ. Sequence context outside the target region influences the effectiveness of miR-223 target sites in the RhoB 3'UTR. Nucleic Acids Res 2010; 38: 239-252.

4 Glorian V, Maillot G, Poles S, et al. HuR-dependent loading of miRNA RISC to the mRNA encoding the Ras-related small GTPase RhoB controls its translation during UV-induced apoptosis. Cell Death Differ 2011; 18: 1692-1701.

Broackes-Carter FC, Mouchel N, Gill D, et al. Temporal regulation of CFTR expression during ovine lung development: implications for CF gene therapy. Hum Mol Genet 2002; 11: 125-131.

Romey MC, Guittard C, Carles S, et al. First putative sequence alterations in the minimal CFTR promoter region. J Med Genet 1999; 36: 263-264.

7 Sinn PL, Anthony RM, McCray PB Jr. Genetic therapies for cystic fibrosis lung disease. Hum Mol Genet 2011; 20: R79-R86.

Hutt DM, Herman D, Rodrigues AP, et al. Reduced histone deacetylase 7 activity restores function to misfolded CFTR in cystic fibrosis. Nat Chem Biol 2010; 6: 25-33.

Ramsey BW, Davies J, McElvaney NG, et al. A CFTR potentiator in patients with cystic fibrosis and the G551D mutation. N Engl J Med 2011; 365: 1663-1672.

Clancy JP, Rowe SM, Accurso FJ, et al. Results of a phase IIa study of VX-809, an investigational CFTR corrector compound, in subjects with cystic fibrosis homozygous for the F508del-CFTR mutation. Thorax 2012; 67: $12-18$.

1 Van Goor F, Hadida S, Grootenhuis PD, et al. Correction of the F508del-CFTR protein processing defect in vitro by the investigational drug VX-809. Proc Natl Acad Sci USA 2011; 108: 18843-18848.

2 Varambally S, Cao Q, Mani RS, et al. Genomic loss of microRNA-101 leads to overexpression of histone methyltransferase EZH2 in cancer. Science 2008; 322: 1695-1699.

Guan P, Yin Z, Li X, et al. Meta-analysis of human lung cancer microRNA expression profiling studies comparing cancer tissues with normal tissues. J Exp Clin Cancer Res 2012; 31: 54 